\title{
Comparing Effectiveness of Different Microhomology Lengths in Microhomology Mediated End Joining (MMEJ) Repair of DNA Double Stranded Breaks (DSBs)
}

\author{
Vasanth Senthilraja ${ }^{1}$, Rehet Chugh ${ }^{1}$, Sehej Chugh ${ }^{2}$, Ethan Yang ${ }^{1}$, Himanshu Wagh ${ }^{3 *}$ \\ ${ }^{1}$ California Northstate University College of Health Sciences, Rancho Cordova, USA \\ ${ }^{2}$ Clovis Unified School District, Fresno, USA \\ ${ }^{3}$ California Northstate University College of Medicine, Elk Grove, USA
}

\begin{abstract}
*Corresponding author: Himanshu Wagh, California Northstate University College of Medicine, Elk Grove, USA, Email: Himanshu.wagh5630@cnsu.edu
\end{abstract}

\section{ABSTRACT}

Keywords

DNA Double-Stranded Breaks (DSBs) are caused by genotoxic agents, such as MMEJ ionizing radiation and chemical agents, and can cause an affected cell to undergo DNA apoptosis or cell death. The process of microhomology-mediated end joining (MMEJ) shows promising results in the repair of DSBs in DNA. MMEJ is a mutagenic DSB repair mechanism that uses a certain length of homologous nucleotides adjacent to the DSB to align the broken DNA strands for repair. This can result in insertions, deletions, and even translocations of genes at the DSB site. This has led to discussions of debate on whether MMEJ is efficient in repairing DSBs in DNA. Based on the length of microhomology, the effectiveness of the DSB repair can vary. The purpose of this research is to examine MMEJ repair using micro-homologies of different lengths in Saccharomyces cerevisiae cells to test the effectiveness of MMEJ repair. The HIS3 gene located in chromosome 15 in the yeast cell is used to test for MMEJ repair, and the full microhomology length represents 311 base pairs (bp). Various crosses are performed on cells to attain desired genotypes that have the homologous chromosomes in alignment for MMEJ repair. After inducing DSBs, media-based testing is used for testing the efficiency of MMEj repair by checking for the presence of certain genes that may have formed or been deleted during the repair process.

\section{INTRODUCTION}

There are many types of damage that can occur to DNA in a cell. They can be divided into two subtypes: endogenous damage caused by reactive oxygen species (ROS) that are the byproducts of metabolic activity and exogenous damage caused by UV, X-ray, gamma radiation, plant toxins, and viruses [1]. One way the cell repairs damaged DNA is by removal of the damaged bases followed by resynthesis of the excised region or by direct reversal of the damage, which may be a more efficient way of dealing with specific types of DNA damage that occur frequently. Only a few types of DNA damage are repaired in this way, particularly pyrimidine dimers resulting from exposure to ultraviolet (UV) light [2]. While direct repair could be a potential way of dealing with particular types of DNA damage, excision repair is a more general means of repairing a wide variety of chemical alterations to DNA [2]. Excision-repair pathways involve removal of the stretch of DNA containing damaged DNA and filling of the resulting gap through DNA replication using the undamaged DNA as a template [3]. Failure to repair DNA defects can cause genomic instability and lead to human genetic diseases that affect a wide 
variety of body systems and a predisposition for cancer. These disorders include ataxia-telangiectasia (AT), a degenerative motor condition caused by failure to repair oxidative damage in the cerebellum, and xeroderma pigmentosum (XP), a condition characterized by sensitivity to sunlight and linked to a defect in an important ultraviolet (UV) damage repair pathway [4]. Studies have also explored genetic causes for diseases like Creutzfeldt Jakob's Disease (CJD) to be initiated by DNA damage [5].

\section{METHODOLOGY}

Several genes were checked for expression during mating crosses to ensure the right genotype was obtained. These genes produce proteins such as leucine (LEU), tryptophan (TRP), uracil (ura), and methionine (met). The first cross that was carried out was between a Mata-inc his3 $\Delta 3^{\prime}(311 \mathrm{bp}$ )HOcs(117bp), ura3::TRP1 genotype and a Mat $\alpha$ his3:NatR LEU2 on YPD media. In all the crosses that were carried out, it was ensured that a Mata cell mated with a Mat $\alpha$ cell as each cannot mate with their own genotype. After the cross was carried out, the diploid cells were selected for by replica plating onto SD- Leu Trp media plates. This helps identify the diploid cells as cells that mated properly and formed diploids would be the only cells that had the genes to grow on the media. The cells that grew were transferred onto sporulation media to induce meiosis. The cells were analyzed for sporulation by placing the cells in a wet microscope slide and analyzing under a microscope for sporulation. Then, tetrad analysis was performed by replica plating the haploid spores onto three different media that were each either Leu-, Trp-, or YPD (agar) with nourseothricin (NAT) antibiotic. The purpose of the tetrad analysis was to find the cells that had the genotype Mat $\alpha$ his $3 \Delta 3$ ' (311 bp) LEU2 by looking for the cells that grew on Leu- media but not on Trp- and YPD with NAT media. These cells had the LEU2 gene to synthesize leucine and survive but lacked the NatR and TRP1 genes to synthesize the associated proteins for survival in the nutrient-deficient media.

The second cross was between the Mat $\alpha$ his $3 \Delta 3$ ' (311 bp) LEU2 and Mata-inc his3::NATR met22::TRP1 rev1::URA3. This helped produce diploid cells that were of the genotype Mata-inc, $\quad \operatorname{his} 3 \Delta 3^{\prime}(311 \quad b p)-H O c s(117 b p)$, met22::TRP1 rev1:URA3. The diploid cells were selected by replica plating onto SD- Leu Trp media plates. These diploid cells were transferred onto sporulation media to induce meiosis and obtain four haploid meiotic product cells/spores. The haploid cells were then plated onto met- TRP- and rev- URA- nutrientdeficient media and analyzed using tetrad analysis to ensure that the correct genotype was obtained. These haploid cells (Mata-inc, his3 $\Delta 3^{\prime}$ (311 bp)-HOcs(117bp), met22::TRP1 rev1:URA3) were then crossed with the genotype Mat $\alpha$-inc his $3 \Delta 5, \operatorname{trp} 1::$ GAL-HOendo::KANMX and grown on YPD media. The diploid cells were selected by replica plating the diploids onto SD- trp media and YPD with Kanamycin (KAN) media. So far, we have carried out all the crosses necessary to achieve the Mata-inc, his3 $\Delta 3$ '-HOcs(117bp), met22::TRP1 rev1:URA3 genotype. These will be crossed with the genotype Mat $\alpha$-inc his3 $\Delta 5$ ' trp $1:: G A L-H O e n d o:$ KANM and then grown in galactose media to induce the DSBs and test the research question of the experiment.

The crossed cells can now be grown in galactose media to activate the HO-endo gene near the GAL promoter that acts as an endonuclease and causes a double-stranded break in chromosome XV. The crossed cells are both Mata-inc or Mat $\alpha$-inc to prevent the endonuclease protein from cleaving the chromosome at the Mata or Mat $\alpha$ site. The ability of the cell to repair the DSB via microhomology-mediated end joining (MMEJ) can be analyzed by transferring the cells from the galactose media to his- media. If the cells had repaired the double-stranded break, then his gene would be intact and allow the cell to grow by synthesizing the His protein. Another way the cell can repair the DSB is by crossing over between each of its homologous XV chromosomes. During the crossover, either the met22::TRP1 genes or rev1::URA3 genes will be lost in the cell that repaired the DSB. These cells can be identified by growing on media that is met- TRPand rev- URA- and looking for cells that have grown on only one of the media types. This would indicate that the cell had repaired the DSB via crossing over and lost one of the genes in the process.

\section{DATA/RESULTS}

Table 1: Cell Growth on Diluted Media and Calculated Recombination Frequency for $311 \mathrm{bp}$

\begin{tabular}{|l|l|l|l|l}
\hline SD His $\left(10^{-4}\right)$ & YPD $\left(10^{-5}\right)$ & His cells & Viable Cells & RF (311 bp)
\end{tabular}

\begin{tabular}{|l|l|l|l|c|}
\hline 237 & 164 & 237 & 1640 & 0.1445122 \\
\hline 220 & 177 & 220 & 1770 & 0.12429379 \\
\hline 172 & 174 & 172 & 1740 & 0.09885057 \\
\hline 183 & 187 & 183 & 1870 & 0.09786096 \\
\hline 225 & 188 & 225 & 1880 & 0.11968085 \\
\hline
\end{tabular}

\begin{tabular}{|l|l|l|}
\hline 311 bp (Median) & 0.11968085 & \\
\hline $\begin{array}{l}\text { Standard } \\
\text { Deviation }\end{array}$ & 0.01944867 & \\
\hline $\begin{array}{l}\text { 95\% Confidence } \\
\text { Interval }\end{array}$ & 0.01739542 & \\
\hline
\end{tabular}


Table 2: Recombination Frequencies and 95\% Confidence Intervals for Different Homologies

\begin{tabular}{|l|c|c|c|c|c|c|}
\hline & $311 \mathrm{bp}$ & $20 \mathrm{bp}$ & $16 \mathrm{bp}$ & $14-2-9 \mathrm{bp}$ & $14-2-4 \mathrm{bp}$ & $14-2-2 \mathrm{bp}$ \\
\hline Median & $1.20 \mathrm{E}-01$ & $6.94 \mathrm{E}-05$ & $1.25 \mathrm{E}-06$ & $3.23 \mathrm{E}-05$ & $5.40 \mathrm{E}-05$ & $9.01 \mathrm{E}-07$ \\
\hline CI Upper Bound & $1.74 \mathrm{E}-02$ & $4.88 \mathrm{E}-06$ & $2.81 \mathrm{E}-07$ & $7.00 \mathrm{E}-05$ & $8.00 \mathrm{E}-05$ & $1.94 \mathrm{E}-07$ \\
\hline CI Lower Bound & $1.74 \mathrm{E}-02$ & $4.88 \mathrm{E}-06$ & $2.81 \mathrm{E}-07$ & $7.00 \mathrm{E}-05$ & $8.00 \mathrm{E}-05$ & $1.94 \mathrm{E}-07$ \\
\hline
\end{tabular}

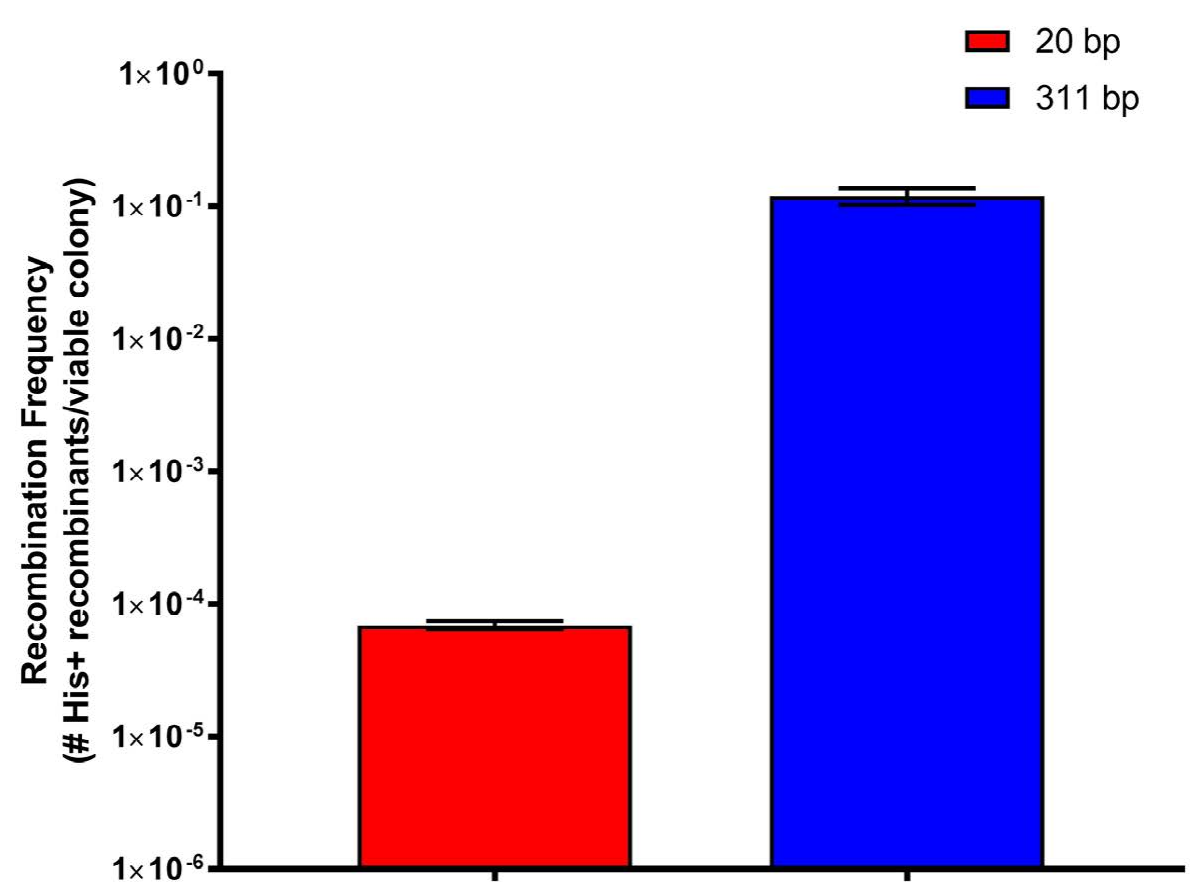

Figure 1: Recombination Frequencies of 311 bp Complete Homology vs 20 bp Microhomology

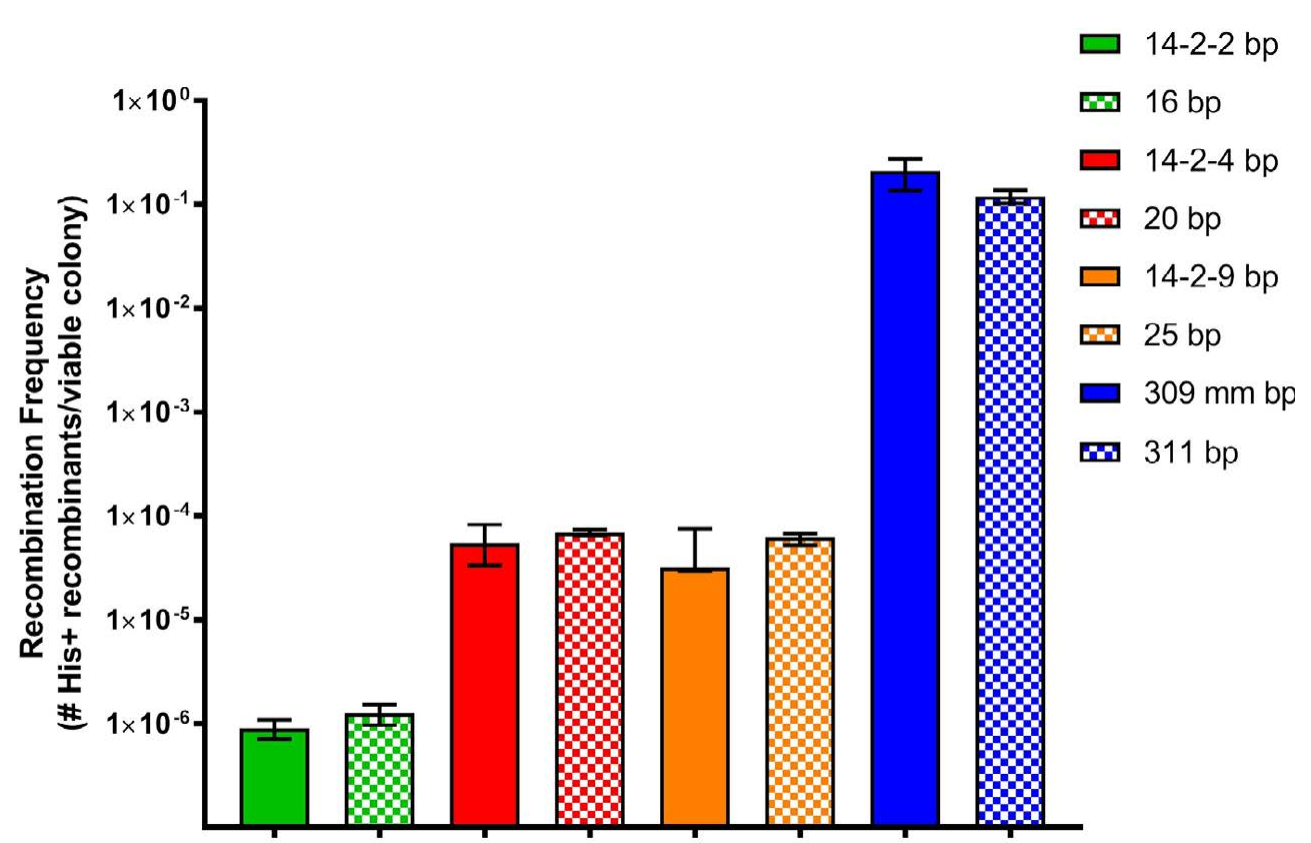

Figure 2: Recombination Frequencies of Multiple Length Homologies 


\section{RESULTS}

Figure 1 lists the median recombination frequencies of the tested 311 base pair (bp) length homologous recombination (HR) repair and the $20 \mathrm{bp}$ length microhomology-mediated end joining (MMEJ) repair mentioned in Table 1. As seen, the HR repair with the complete homology length had the greater recombination frequency of $1.1^{*} 10^{\wedge}-1$ and was the most efficient at repairing double-stranded breaks (DSBs) in DNA. The MMEJ repair had a smaller recombination frequency median value of $6.9^{*} 10^{\wedge}-5$ and wasn't as efficient at repairing the DSB compared to HR. The recombination frequency values were tested using a $95 \%$ confidence interval for accuracy and validity. The reason for a greater recombination frequency and repair rate in HR can be attributed to the fact that HR has a greater homology length of $311 \mathrm{bp}$ compared to $20 \mathrm{bp}$ in MMEJ. Furthermore, the process of MMEJ is also more error-prone than HR, due to the genomic instabilities, such as chromosome translocations, that occur in MMEJ. This is believed to have led to a diminished recombination frequency rate in the cells that used MMEJ to repair DSBs.

Figure 2 lists the recombination frequencies of different base pair lengths of MMEJ repair and HR repair in Table 2. As seen in the graph, the HR repair (311 bp and $309 \mathrm{bp}$ ) overall had greater recombination frequencies than the 14, 16, 20, and 25 base-pair length MMEJ repairs, with and without deletions. This is due to the higher susceptibility of MMEJ to errors in DSB repair compared to HR. Among the different MMEJ base pair lengths, the homologies without deletions had greater recombination frequencies than the homologies with deletions. This can be attributed to the fact the two base-pair length deletions disrupted the efficiency of the MMEJ repair, leading to lower recombination frequencies. Among the 14 base pair homologies, as the two base pair deletion occurred more in the center of the homology (14-2-9 bp) compared to the ends of the homology (14-2-2 and 14-2-4) strands, the recombination frequency decreased. This is due to the fact that a deletion in the center of the homology length affects the mechanism of the MMEJ repair to a greater extent than at the end of the homology lengths.

\section{DISCUSSION}

Double-Stranded Breaks (DSBs) are one of the most dangerous types of DNA damage and are complicated to repair through normal cell repair methods, such as base-pair removal or excision repair. The two known methods for repairing DSBs are homologous recombination (HR) and non-homologous end-joining (NHEJ) [6]. In HR, the DNA ends are first resected in the $5^{\prime}$ to $3^{\prime}$ direction by nucleases, and the resulting 3 single-stranded tails then invade the DNA double helix of a homologous, undamaged partner molecule [7]. They are then extended by the action of DNA polymerase, which copies information from the partner [7]. Following branch migration, the resulting DNA crossovers (Holliday junctions) are resolved to yield two intact DNA molecules [7]. HR usually has a length anywhere in between 30-200 nucleotides. In NHEJ, the DSB is first recognized by the $\mathrm{Ku} 70-\mathrm{Ku} 80$ heterodimer $(\mathrm{Ku})$, which acts as a loading protein to which other NHEJ proteins can be recruited to promote the joining of DNA ends [8]. While HR is a rather error-free process, it requires large nucleotide lengths of DNA that are not always available. A limitation of NHEJ is that it is reliant on the $\mathrm{Ku}$ proteins for DNA end-joining and can lead to loss or gain of DNA information in the process [9]. While MMEJ is known to be more error-prone than both HR and NHEJ, it does not rely on the $\mathrm{Ku} 70 / \mathrm{Ku} 80$ proteins and relies on micro-homologies that are 2-20 nucleotides long.

Micro-homologies are short repetitive segments of DNA that could be potential sites of rearrangement in different types of cancer. Hence, it is important to understand how chromosome rearrangement occurs within DNA micro-homologies and what genes play a role in the process. As the MMEJ pathway is upregulated in cancers, the genes and proteins involved in MMEJ can be targeted for cancer therapy [10]. In addition, the Human Papilloma Virus (HPV) has been identified in using MMEJ pathways and can be targeted for certain drug therapies [11]. Our research focused on testing the efficacy of MMEJ repair pathways based on different microhomology lengths and discovered that there is a positive correlation between microhomology length and MMEJ repair effectiveness.

The purpose of this research was to determine the efficacy of MMEJ repair based on different micro-homological lengths. These segments of micro-homologies are locations of potential sites where rearrangement occurs that may result in cancer [12-23]. Thus, it is important to understand the effectiveness of MMEJ repair based on different micro-homological lengths. The eukaryotic species used to test MMEJ repair in this experiment is the yeast cell Saccharomyces cerevisiae. This organism contains the components and genes necessary for testing MMEJ repair through several mating crosses and inducing DSBs. By measuring the number of viable cells after inducing double-stranded breaks, the relationship between microhomology lengths and MMEJ repair effectiveness can be determined. Nevertheless, further research is needed in order to learn more about MMEJ repair. Perhaps trying therapies with different compounds such as EDTA would yield different results, or maybe different AI software could be developed to help process the lab results from recombination at a faster rate [24-30]. The findings from this paper could help scientists gain a more thorough understanding of diseases such as CJD 
and psoriasis [5, 24]. Future studies should also be conducted to ascertain why longer homologies are more efficient at repairing DSBs.

\section{CONCLUSION}

The research focused on examining the effectiveness of MMEJ repair of DBSs in DNA using micro-homologies of different lengths in yeast cells. Based on the results obtained from the experiment, it can be understood that the longer the length of the homology involved in the DSB repair, the higher the recombination frequency. This could be primarily due to the fact that a longer homology allows for more efficient repair without any chromosomal rearrangements occurring in the process. Hence, the longer, complete homologies (311 bp and $309 \mathrm{bp}$ ) were able to undergo HR to repair the DSBs with higher recombination frequencies, whereas shorter microhomologies contained multiple errors that caused them to have smaller cell survival numbers and lower recombination frequencies. Deletions in micro-homologies that were more in the central region of the chromosomes than in the ends of the chromosome reduced the recombination frequency of the micro-homologies to a greater extent. Overall, longer, more complete homologies were more efficient at repairing DSBs in DNA than shorter micro-homologies of DNA in chromosomes.

\section{REFERENCES}

1. Shimada, K., Crother, T. R., \& Arditi, M. (2014). DNA Damage Responses in Atherosclerosis. Biological DNA Sensor, 231-253. doi:10.1016/b978-0-12-404732-7.00010-1

2. Cooper GM. The Cell: A Molecular Approach. 2nd edition. Sunderland (MA): Sinauer Associates; 2000. DNA Repair. Available from: https://www.ncbi.nlm.nih.gov/books/NBK9900/

3. Izumi, T., \& Mellon, I. (2016). Base Excision Repair and Nucleotide Excision Repair. Genome Stability, 275-302. doi:10.1016/b978-0-12-803309-8.00017-3

4. Clancy, S. (2008) DNA damage \& repair: mechanisms for maintaining DNA integrity. Nature Education 1(1):103

5. Senthilraja, V., Lou, E., Nakka, A., Karamian, P., Aslam, I., Rabara, J., Gahoonia, N., Kaur, N., Reddy, A., Ber, T., \& Wagh, H. (2021). Exploring drug and antibody-based treatment options for creutzfeldt-jakob disease. Applied Cell Biology, 9(2), 43-48. https://doi.org/10.53043/2320-1991.acb90008

6. Haber, J.E. Partners and pathways repairing a double-strand break. Trends Genet. 16, 259-264 (2000).

7. Khanna, K. K., \& Jackson, S. P. (2001). DNA double-strand breaks: signaling, repair and the cancer connection. Nature Genetics, 27(3), 247-254.doi:10.1038/85798

8. Chang, H. H. Y., Pannunzio, N. R., Adachi, N., \& Lieber, M. R. (2017). Non-homologous DNA end joining and alternative pathways to double-strand break repair. Nature Reviews Molecular Cell Biology, 18(8), 495-506.doi:10.1038/nrm.2017.48
9. Seol, J.-H., Shim, E. Y., \& Lee, S. E. (2018). Microhomologymediated end joining: Good, bad and ugly. Mutation Research/ Fundamental and Molecular Mechanisms of Mutagenesis, 809, 81-87. doi:10.1016/j.mrfmmm.2017.07.0

10. Patterson-Fortin, J., \& D'Andrea, A. D. (2020). Exploiting the Microhomology-Mediated End-Joining Pathway in Cancer Therapy. Cancer Research, canres.1672.2020. doi:10.1158/00085472.can-20-1672

11. Leeman, J. E., Li, Y., Bell, A., Hussain, S. S., Majumdar, R., RongMullins, X., ... Higginson, D. S. (2019). Human papillomavirus 16 promotes microhomology-mediated end-joining. Proceedings of the National Academy of Sciences, 201906120.doi:10.1073/ pnas. 1906120116

12. McVey M, Lee SE. MMEJ repair of double-strand breaks (director's cut): deleted sequences and alternative endings. Trends Genet. 2008 Nov;24(11):529-38. doi: 10.1016/j.tig.2008.08.007

13. Seol JH, Shim EY, Lee SE. Microhomology-mediated end joining: Good, bad and ugly. Mutat Res. 2018 May;809:81-87. doi: 10.1016/j.mrfmmm.2017.07.002

14. Sfeir A, Symington LS. Microhomology-Mediated End Joining: A Back-up Survival Mechanism or Dedicated Pathway? Trends Biochem Sci. 2015 Nov;40(11):701-714. doi: 10.1016/j. tibs.2015.08.006

15. McVey M, Lee SE. MMEJ repair of double-strand breaks (director's cut): deleted sequences and alternative endings. Trends Genet. 2008 Nov;24(11):529-38. doi: 10.1016/j.tig.2008.08.007

16. Seol JH, Shim EY, Lee SE. Microhomology-mediated end joining: Good, bad and ugly. Mutat Res. 2018 May;809:81-87. doi: 10.1016/j.mrfmmm.2017.07.002

17. Yanik M, Ponnam SPG, Wimmer T, Trimborn L, Müller C, Gambert I, Ginsberg J, Janise A, Domicke J, Wende W, Lorenz B, Stieger K. Development of a Reporter System to Explore MMEJ in the Context of Replacing Large Genomic Fragments. Mol Ther Nucleic Acids. 2018 Jun 1;11:407-415. doi: 10.1016/j. omtn.2018.03.010

18. Kostyrko K, Mermod N. Assays for DNA double-strand break repair by microhomology-based end-joining repair mechanisms. Nucleic Acids Res. 2016 Apr 7;44(6):e56. doi: 10.1093/nar/gkv1349

19. Sinha S, Villarreal D, Shim EY, Lee SE. Risky business: Microhomology-mediated end joining. Mutat Res. 2016 Jun;788:17-24. doi: 10.1016/j.mrfmmm.2015.12.005

20. Truong LN, Li Y, Shi LZ, Hwang PY, He J, Wang H, Razavian N, Berns MW, Wu X. Microhomology-mediated End Joining and Homologous Recombination share the initial end resection step to repair DNA double-strand breaks in mammalian cells. Proc Natl Acad Sci U S A. 2013 May 7;110(19):7720-5. doi: 10.1073/ pnas. 1213431110

21. Wang $\mathrm{H}, \mathrm{Xu} \mathrm{X}$. Microhomology-mediated end joining: new players join the team. Cell Biosci. 2017 Jan 13;7:6. doi: 10.1186/ s13578-017-0136-8

22. Khodaverdian VY, Hanscom T, Yu AM, Yu TL, Mak V, Brown AJ, Roberts SA, McVey M. Secondary structure forming sequences drive SD-MMEJ repair of DNA double-strand breaks. Nucleic Acids Res. 2017 Dec 15;45(22):12848-12861. doi: 10.1093/nar/gkx1056 


\section{Research Article}

23. Patterson-Fortin J, D'Andrea AD. Exploiting the MicrohomologyMediated End-Joining Pathway in Cancer Therapy. Cancer Res. 2020 Nov 1;80(21):4593-4600. doi: 10.1158/0008-5472.CAN20-1672

24. Sunil A, Shaheed G, Reddy AJ, Nawathey N, Brahmbhatt H: A review on the role of ethylenediaminetetraacetic acid (EDTA) in the treatment and understanding of psoriasis. Cureus. 2021, 16:e16424. 10.7759/cureus.16424

25. Reddy AJ, Tak N, Martel JB: Case study of endogenous streptococcal endophthalmitis in the critical care setting. Cureus. 2021, 5:e16192. 10.7759/cureus.16192

26. Wagh H, Reddy AJ: A comparison of DASH scores resulting from different treatment options for the intra-articular distal radius fracture in the geriatric population. Appl Cell Biol. 2021, 9:18-23. 10.53043/2320-1991.acb90004
27. Tak N, Reddy A J, Martel J, et al. Clinical Wide-Field Retinal Image Deep Learning Classification of Exudative and NonExudative Age-Related Macular Degeneration. Cureus 13(8): e17579. doi:10.7759/cureus.17579

28. Tak N, Wagh P, Sandhu S, Reddy A, Wagh H: A quantitative analysis on the effect of varying nitrate concentrations on $\mathrm{pH}$ levels on the growth of algae. Appl Cell Biol. 2021, 9:24-28. 10.53043/2320-1991.acb90005

29. Reddy AJ, Martel JB: Deep neural network learning for detection and grading of diabetic retinopathy. Appl Cell. 2020, 8:67-73. 10.53043/2320-1991.acb90001

30. Sandhu S, Sandhu A, Reddy AJ, Wagh H, et al. :An Investigative Study into the C. flumenia and its Interactions with its Surrounding Ecosystem. Appl Cell Biol. 2021, 9:33-42. 10.53043/2320-1991. acb90007 